\title{
Backreaction of tracer particles on vortex tangle in helium II counterflow
}

\author{
E. Varga - C. F. Barenghi · Y. A. \\ Sergeev • L. Skrbek
}

\begin{abstract}
We report computer simulations of the interaction of seeding particles with quantized vortices and with the normal fluid flow in thermal counterflow of superfluid ${ }^{4} \mathrm{He}$. We show that if the particle concentration is too large, the vortex tangle is significantly affected, posing problems in the interpretation of visualization experiments. The main effects are an increase in vortex line density and a change in polarization of the vortex tangle, caused by the action of the Stokes drag of the viscous normal fluid on the trapped particles. We argue that in the case of large particle concentration, typically used for the particle image velocimetry technique, the tangle properties might become significantly changed. On the contrary, the particle tracking velocimetry technique that uses smaller particle concentration should not be appreciably affected.
\end{abstract}

\section{Introduction}

Flow visualization is one of the most valuable experimental tools in fluid dynamics. A variety of techniques exists which seed the fluid with contrasting agent that can be easily observed, e.g., by a sensitive camera 1]. Visualization techniques have already been used at low temperatures to study both classical and quantum cryogenic flows, even though their application is difficult, for various technical reasons, such as optical access to the experimental volume or

E. Varga, L. Skrbek

Faculty of Mathematics and Physics, Charles University, Ke Karlovu 3, Prague, Czech Republic

E-mail: varga.emil@gmail.com

C. F. Barenghi

Joint Quantum Centre Durham-Newcastle, School of Mathematics and Statistics, Newcastle University, Newcastle upon Tyne NE1 7RU, United Kingdom

Y. A. Sergeev

Joint Quantum Centre Durham-Newcastle, School of Mechanical and Systems Engineering, Newcastle University, Newcastle upon Tyne NE1 7RU, United Kingdom 
choice of suitable seeding particles. As for quantum flows and turbulence [2] (so far of superfluid ${ }^{4} \mathrm{He}$, known as He II), application of visualization techniques face additional fundamental difficulties, due to the existence of two velocity fields and the interaction of seeding particles with quantized vortices 3 . Despite these problems, the implementation of visualization methods in He II has led to the direct visualization of quantized vortices in [4] and important results [5] about Kelvin waves [6] and vortex reconnections [7], vortical structures around a cylinder 8, non-Gaussian velocity statistics ,9, 10, and non-classical acceleration statistics 11, crossover between quantum and classical behaviour [10,12]; particles' trapping mechanisms [13], and added mass effects [1]. In comparison with classical fluid dynamics, visualization of quantum flows is not yet firmly established and the interpretation of experiments poses important fundamental questions.

The questions arise because He-II differs from classical liquids in several important aspects [2]. For $1 \mathrm{~K}<T<T_{\lambda}$, where visualization methods are usually applied, He-II can be described as consisting of two fluids - the inviscid superfluid component carrying no entropy, and the viscous normal fluid behaving approximately as an ordinary Navier-Stokes fluid. Turbulence in the superfluid component can only exist in the form of a complex tangle of quantized vortices - thin topological defects around which the circulation is restricted to single quantum of circulation $\kappa=h / M$ where $h$ is the Planck constant and $M$ is the mass of ${ }^{4} \mathrm{He}$ atom.

This complex nature of He-II poses challenges for the interpretation of visualization experiments. Here we consider potential problems that might occur in the interpretation of particle tracking velocimetry (PTV) and/or particle image velocimetry (PIV) techniques, both relying on observing seeding particles (in recent experiments mostly solid hydrogen or deuterium flakes [5]) suspended in the flow. These particles interact with both the normal and superfluid velocity fields 3, 5, 11 and can become trapped on the cores of quantized vortices. However, in most cases they are treated as non-intrusive, passive probes of the flow under study. It is therefore of great interest to try to assess the degree of non-ideality of the particles, which might lead to a distorted physical information about the quantum flow under study and, consequently, to a misleading conclusion on some important aspects of quantum turbulence.

To this end, we perform a series of numerical experiments, extending the work of Mineda et al. 14, which simulates the movement of seeding particles in the velocity field due to the counterflow tangle of quantized vortices. We find that a trapped particle deforms the vortex on which it is trapped, stretching it in the direction of the flowing normal fluid, via the action of the Stokes drag. A large number of particles can increase the vortex line density by up to $100 \%$ and change its polarization. We compare these more realistic results with those obtained by modelling trapped particles as ideal tracers of the vortices and find significant differences, both in the probability of trapping and the velocity statistics. 


\section{Computational setup and results}

We perform a vortex filament simulation of counterflowing He-II in periodic boundary conditions. The normal fluid velocity $\boldsymbol{v}_{n}$ is uniform and statically prescribed. To a statistically converged counterflow tangle, we add inertial particles at random positions and initially zero velocities. The movement of both vortices and particles is described below.

The quantized vortices in He-II are modelled, following the seminal work of Schwarz [15], as one-dimensional spatial curves of arbitrary shape. These vortex lines (labelled $s(\xi)$, where $\xi$ is the arc length along the line) induce a superfluid velocity $\boldsymbol{v}_{s}$ given by the standard [15, 16, 17] Biot-Savart integral

$$
\boldsymbol{v}_{s}(\boldsymbol{r})=\frac{\kappa}{4 \pi} \oint_{\mathcal{L}} \frac{\boldsymbol{s}^{\prime}(\xi) \times[\boldsymbol{r}-\boldsymbol{s}(\xi)]}{|\boldsymbol{r}-\boldsymbol{s}(\xi)|^{3}} \mathrm{~d} \xi
$$

with $\mathcal{L}$ denoting the entire configuration of lines in the vortex tangle. The movement of the vortices themselves is determined from the balance of forces, namely Magnus force and mutual friction, under the assumption that the vortices are massless (e.g., [14). The resulting equation of motion is

$$
\dot{\boldsymbol{s}}=\boldsymbol{v}_{s}^{\prime}(\boldsymbol{s})+\alpha \boldsymbol{s}^{\prime} \times\left(\boldsymbol{v}_{n}-\boldsymbol{v}_{s}\right)+\alpha^{\prime} \boldsymbol{s}^{\prime} \times\left[\boldsymbol{s}^{\prime} \times\left(\boldsymbol{v}_{n}-\boldsymbol{v}_{s}\right)\right],
$$

where the dot denotes the time derivative and the prime denotes derivative with respect to the arc-length. The prime on the superfluid velocity in (2) denotes the standard de-singularization of the Biot-Savart integral

Particle dynamics. Several past studies were concerned with modelling inertial particles that are either far away the from cores of the quantized vortices [18,3] or remain trapped on them [14]. Studies that address the full range of possible interactions of finite spherical particles and quantized vortex lines have already been performed [19], however, the computational complexity of the methods used there prevents the scaling of the simulation to high densities of vortex tangle and/or high number of particles.

In the present study we adopt a mixed approach. Unlike [19, particles are considered as point-like objects. Particles sufficiently far from the vortex lines are considered free and interact with vortices only through inertial forces following 18, such free particles interact with both the normal and superfluid component inertially and with normal component also viscously so that the equation of motion for the the free particles' velocity $\boldsymbol{v}_{p}$ is

$$
\frac{\mathrm{d} \boldsymbol{v}_{p}}{\mathrm{~d} t}=\frac{\rho_{s}}{\rho} \frac{\mathrm{D} \boldsymbol{v}_{s}}{\mathrm{D} t}+\frac{\rho_{s}}{\rho} \frac{\mathrm{D} \boldsymbol{v}_{n}}{\mathrm{D} t}-\frac{\boldsymbol{v}_{p}-\boldsymbol{v}_{n}}{\tau},
$$

where $\mathrm{D} \boldsymbol{v} / \mathrm{D} t=\partial \boldsymbol{v} / \partial t+(\boldsymbol{v} \cdot \nabla) \boldsymbol{v}$ and $\tau=2 a^{2} \rho / 9 \mu_{n}$ is the viscous relaxation time, with $\rho$ being the density of fluid or the particles (particles are assumed to be neutrally buoyant), $a$ is the radius of the particles and $\mu_{n}$ denotes the dynamic viscosity of the normal fluid component. Time-independent and uniform normal fluid velocity is used in the present simulations, therefore the second term on the right-hand side of (3) is identically zero. 
Upon sufficiently close approach of a particle to a vortex, the particle can become trapped. Trapped particle experiences additional forces acting on it namely the vortex tension, the Magnus force and the mutual friction [14. It should be noted, however, that the particles are still assumed to be infinitesimally small and therefore a particle trapped on a vortex does not modify the superfluid velocity induced by the vortex, except possibly deforming the vortex itself. The modified equation of motion for the trapped particles becomes

$$
\begin{aligned}
\frac{\mathrm{d} \boldsymbol{v}_{p}}{\mathrm{~d} t} & =\frac{\boldsymbol{v}_{n}-\boldsymbol{v}_{p}}{\tau}+\frac{\rho_{s}}{\rho} \frac{\mathrm{D} \boldsymbol{v}_{s}^{\prime}}{\mathrm{D} t} \\
& +\left\{T_{0}\left(\boldsymbol{s}_{+}^{\prime}-\boldsymbol{s}_{-}^{\prime}\right)+\rho_{s} \kappa \boldsymbol{s}^{\prime} \times\left(\boldsymbol{v}_{p}-\boldsymbol{v}_{s}\right)\right. \\
& \left.+\left(\gamma_{0} \boldsymbol{s}^{\prime} \times\left[\boldsymbol{s}^{\prime} \times\left(\boldsymbol{v}_{p}-\boldsymbol{v}_{n}\right)\right]+\gamma_{0}^{\prime} \boldsymbol{s}^{\prime} \times\left(\boldsymbol{v}_{p}-\boldsymbol{v}_{n}\right)\right) \Delta \xi\right\} \frac{1}{M_{e f f}}
\end{aligned}
$$

where $T_{0}=\rho_{s} \kappa^{2} / 4 \pi \log \left[2 \sqrt{l_{+} l_{-}} / \sqrt{e} \xi_{0}\right]$ is the vortex tension (energy of the vortex per unit length) with $l_{+}, l_{-}$being the distances to the neighbouring discretisation points along the line and $\xi_{0} \approx 1 \AA$ being the vortex core parameter, $\gamma_{0}$ and $\gamma_{0}^{\prime}$ are mutual friction parameters related to the more standard $\alpha$ and $\alpha^{\prime}$ 20], $\boldsymbol{s}_{-}^{\prime}$ and $\boldsymbol{s}_{+}^{\prime}$ are right and left tangents of the vortex line (the line could be non-smooth at the site where the particle is trapped); $s^{\prime}$ is the tangent at the trapping site calculated as if the line were smooth, $M_{\text {eff }}=3 / 2 \rho V_{p}$ is the effective mass of the particle ( $V_{p}$ being its volume) and $\Delta \xi$ is the maximum discretisation distance along the vortex, $1.6 \times 10^{-5} \mathrm{~m}$.

From the point of view of the vortex, the equation of motion is changed from (2) for the single discretisation point that hosts the trapped particle, to (41). Even though the equation of motion for only a single point is changed, this affects the local geometry of the line thus affecting the neighbourhood of this point as well.

The present work differs from that of Mineda et. al [14] in that we consider both free and trapped particles simultaneously. This requires that the particles, once trapped, can de-trap and become free again. Algorithmically, the particles de-trap only if they are attached to a small vortex loop that is annihilated as a part of small-scale numerical cutoff. This mechanism roughly corresponds to a physical scenario (see Fig. 1a) where a vortex loop collapses into the surface of the particle. An additional scenario of de-trapping is also captured (see Fig. 1b) - the trapped particle produces a cusp on the line sharp enough to cause reconnection producing a small loop that is immediately annihilated.

This treatment of trapped particles is compared with the limiting case of particle so light that they do not affect the movement of the vortices in any way. In this case the particle is simply attached to a discretisation point along the vortex line and follows its movement - that is, its movement is described by (2). For the lack of a better name, this case will be henceforth referred to as "ideal" particle and the case described above as "non-ideal".

We performed several simulations with various numbers of particles $N_{p}$ $(100,250,500,750$, and 1000$)$ in a $1 \mathrm{~mm}^{3}$ cubic computational box with periodic boundary conditions. All particles experience Stokes drag corresponding 

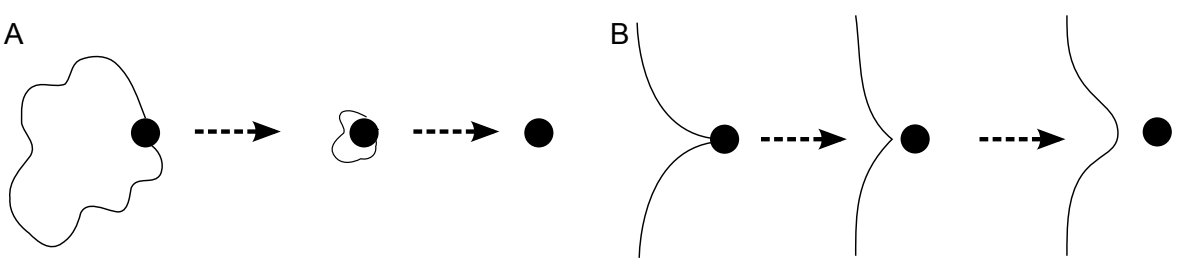

Fig. 1 Two mechanisms of de-trapping

to a sphere with $5 \mu \mathrm{m}$ radius. The imposed flow condition was counterflow with uniform, stationary normal fluid velocity $v_{n}=0.55 \mathrm{~cm} / \mathrm{s}$ and stationary imposed superfluid velocity. The initial condition of each simulation was a vortex tangle converged to a statistically stationary state with total vortex line length of about $6.7 \mathrm{~cm}$. The initial condition for the particles was zero velocity and random position, all the particles being free. All necessary parameters were taken from [21] at $\mathrm{T}=1.9 \mathrm{~K}$.

After introducing the particles into the tangle, we observe two major changes in its properties. Firstly, as can be seen in Fig. 2, the particles increase the tangle density and for the highest number of particles this increase is nearly $100 \%$. Secondly, the polarization of the tangle is also affected. Counterflow tangles are always partially polarized in the sense that the vortex line length in the transverse direction is larger than in the stream-wise direction, due to expansion of the favourably oriented vortex loops by mutual friction. Introduction of the particles is seen, in Fig. 2, to decrease this anisotropy that is, to increase the relative length of the vortex tangle projected onto the stream-wise direction.

Statistical properties of the particles' motion, in the form of probability density functions (PDFs) of the instantaneous velocity component of free and trapped particles, are shown in Fig. 3 for both non-ideal and ideal particles. In Fig. 4, the percentage of the trapped particles is also shown. Neither free nor trapped particles significantly dominate and, indeed, the PDFs feature contributions from both. The narrow peaks correspond to the uniform motion of the free particles determined mostly by the Stokes drag. The wide background distribution is a result of more chaotic movement of the trapped particles.

\section{Discussion and Conclusions}

Flow visualization using frozen hydrogen/deuterium particles is an important experimental tool of contemporary quantum turbulence research. Understanding the detailed behaviour of the particles is therefore of utmost importance for extracting information from the particles' observed movement. Since detailed numerical simulation such as Ref. [19] is at this point prohibitively computationally expensive for large number of particles and/or dense tangles, our attempt at providing some insight into particle dynamics is through the sim- 


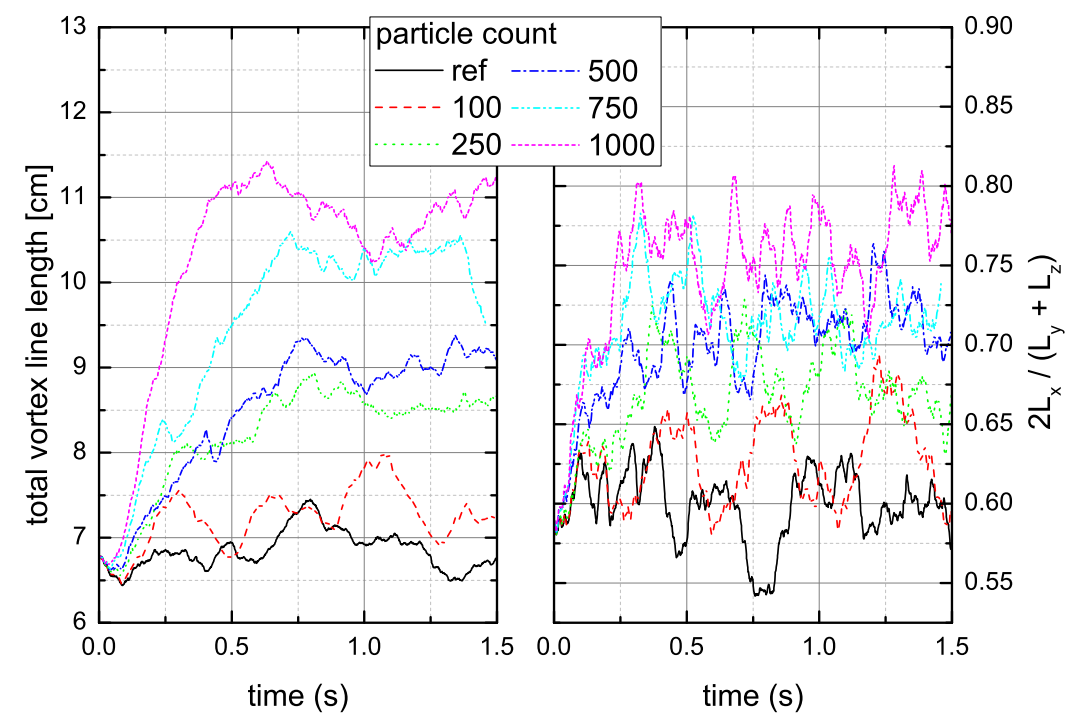

Fig. 2 (Left) Total vortex line length in $1 \mathrm{~mm}^{3}$ periodic computational box for different particle number densities. (Right) Polarization of the vortex tangle, shown here as a ratio of the vortex length projected in stream-wise direction $(x)$ to that in transverse direction (mean of $y$ and $z$ ).
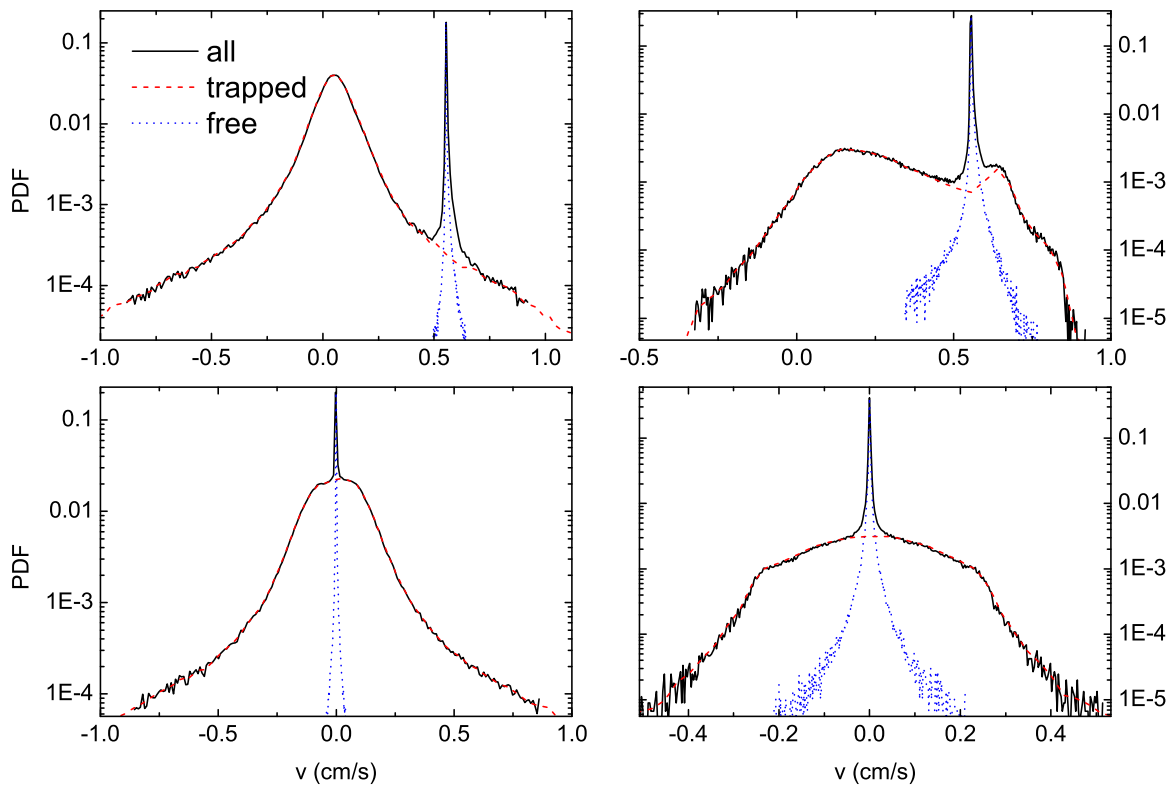

Fig. 3 Probability density functions of particles' velocity component, for both non-ideal (right) and ideal (left) particles. Stream-wise (first row) and transverse (second row) components are shown. 


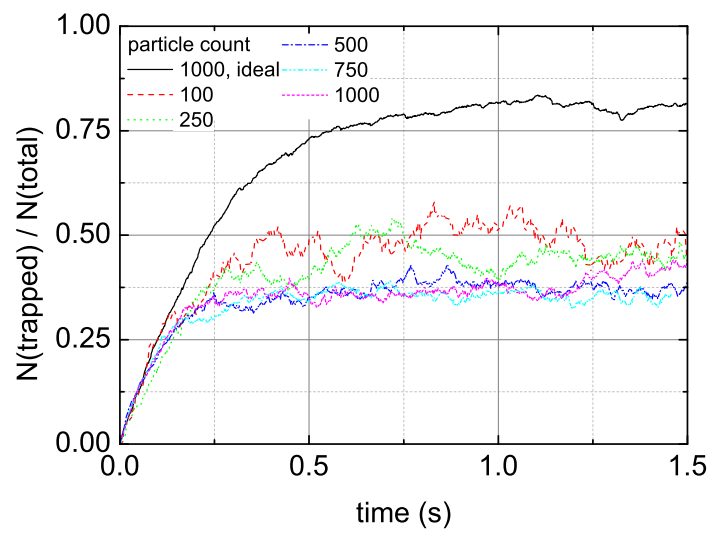

Fig. 4 The percentage of trapped particles. Notice that the vortex stretching caused by the particles decreases the number of trapped particles by nearly a half. Notice also that the final ratio of trapped particles is nearly independent of the total number of particles, meaning that global changes to the structure of the tangle do not significantly affect de-trapping.

plified point-like model used by Mineda et al. 14, but including trapping and de-trapping of the particles on the cores of quantized vortices.

Our data show that the intricate interaction of the particles with quantized vortices can indeed become significant, and particles can cease to act as passive probes. This is evidenced by a significant increase in the vortex line density and by a change of the polarization of the tangle (Fig. 2). These global tangle properties, however, are significantly affected for high enough particle concentration only. Careful particle tracking velocimetry (PTV) experiments that use low particle concentrations (typically about $1 \mathrm{~mm}^{-3}$ ) should not be appreciably affected. Results of methods that use higher particle concentration, such as particle image velocimetry (PIV), should be interpreted with care.

Moreover, one should remain cautious even if the low particle concentration does not affect global mean properties of the tangle. From the trapping rate (Fig. 4) one can see that both trapped and free particles give significant contributions to the overall statistical properties of the particle motion. Trapped particles, however, do not sample the vortex as it would have moved without this trapped particle - it locally deforms it and causes it to move with slightly artificial velocity.

The effect of the back-interaction of the particles on the vortices on the statistics of the particles' velocities can be seen in Fig. 3. It should be noted that the sharp peak corresponding to the free particles in the PDFs is generally not observed in the experiments. The most probable causes for this discrepancy, besides the generally wide distribution of particles sizes and shapes, is that the normal fluid component in the experiments is turbulent, while in the simulations it is laminar.

The program used in the simulations is a modified version of the qvort code created by Andrew Baggaley and others, to whom we are grateful. The 
research is financially supported by the Charles University in Prague under GAUK No. 366213.

\section{References}

1. M. Raffel, C.E. Willert, S.T. Werely, J. Kompenhans, Particle Image Velocimetry - A Practical Guide (Springer, Berlin, 2007)

2. C.F. Barenghi, L. Skrbek, K.R. Sreenivasan, Proc. Natl. Acad. Sci. U.S.A. 111, 4647 (2014)

3. Y.A. Sergeev, C.F. Barenghi, Journal of Low Temperature Physics 157(5-6), 429 (2009)

4. G.P. Bewley, D.P. Lathrop, K.R. Sreenivasan, Nature 441(7093), 588 (2006)

5. W. Guo, M. La Mantia, D.P. Lathrop, S.W. Van Sciver, Proc. Natl. Acad. Sci. U.S.A. 111, $4653(2014)$

6. E. Fonda, D.P. Meichle, N.T. Ouellette, S. Hormoz, D.P. Lathrop, Proc. Natl. Acad. Sci. U.S.A. 111, 4707 (2014)

7. M. Paoletti, M.E. Fisher, D.P. Lathrop, Physica D 239(14), 1367 (2010)

8. T. Zhang, S.W. Van Sciver, Nature Physics 1(1), 36 (2005)

9. M.S. Paoletti, M.E. Fisher, K.R. Sreenivasan, D.P. Lathrop, Phys. Rev. Lett. 101(15), $154501(2008)$

10. M. La Mantia, L. Skrbek, EPL (Europhysics Letters) 105(4), 46002 (2014)

11. M. La Mantia, L. Skrbek, Physical Review B 90(1), 014519 (2014)

12. A.W. Baggaley, C.F. Barenghi, Physical Review E 84(6), 067301 (2011)

13. T. Chagovets, S. Van Sciver, Phys. Fluids 23(10), 107102 (2011)

14. Y. Mineda, M. Tsubota, Y.A. Sergeev, C.F. Barenghi, W.F. Vinen, Phys. Rev. B 87(17), $174508(2013)$

15. K.W. Schwarz, Phys. Rev. B 38, 2398 (1988)

16. A.W. Baggaley, C.F. Barenghi, Physical Review B 83(13), 134509 (2011)

17. H. Adachi, S. Fujiyama, M. Tsubota, Phys. Rev. B 81, 104511 (2010)

18. D.R. Poole, C.F. Barenghi, Y.A. Sergeev, W.F. Vinen, Phys. Rev. B 71(6), 064514 (2005)

19. D. Kivotides, C.F. Barenghi, Y.A. Sergeev, Phys. Rev. B 77, 014527 (2008)

20. R.J. Donnelly, Quantized Vortices in Helium II (Cambridge University Press, 1991)

21. R.J. Donnelly, C.F. Barenghi, J. Phys. and Chem. Ref. Data 27, 1217 (1998) 